\title{
Editorial
}

\section{Molecular Modeling: Theory and Applications}

\author{
Medhat A. A. Ibrahim,§ \\ Spectroscopy Department, National Research Centre, 12311, Dokki, Cairo, Egypt
}

Molecular modeling aims to enhance the communication between experimental research and computational work on both existing and new advanced materials. Recently computational methods, have been seen in rapid growth. The continuous improvements in computational methods software have allowed the researchers to apply their theories to molecular systems of unprecedented size and at levels of theory that were simply intractable only a few years ago. Accordingly, The Open Spectroscopy Journal is launching this issue on "Molecular Modeling: Theory and Applications". It gives me great pleasure to present this collection of 3 papers for this issue.

The first paper is performing PM3 quantum mechanical calculations to study the possible polymerization of aniline on the surface of fullerene. Polymerization is tested with fullerene and fulleropyrrolidine surfaces. The polymerized anilines on both fullerene and fulleropyrrolidine are stable while band gap energy indicates that polyaniline which polymerizes on fullerene is the most probable. Both experimental and higher levels of theory at B3LYP confirms the suitability of the quantum mechanical calculations for this work.

\footnotetext{
*Address correspondence to this author at the Spectroscopy Department, National Research Centre, 12311, Dokki, Cairo, Egypt:

Tel: +20122 2727636; Fax:+202 33370931;

E-mail: medahmed6@yahoo.com

${ }^{\S}$ Guest Editor
}

Second paper presents binary lead borate glass system doped with minor quantities of copper. Deconvolution analysis DAT combined with DFT technique were employed to interpret both theoretical and experimental IR data of this glass system. Experimental IR data reveal the presence of both triangular and tetrahedral borate groups besides the sharing of $\mathrm{Pb}-\mathrm{O}$ units. Undoped glass sample is observed to exhibit strong UV absorption due to the combined contributions of absorption of both $\mathrm{Pb}^{2+}$ ions and trace iron impurities. The presence of both $\mathrm{Cu}^{2+}$ and $\mathrm{Cu}^{+}$has been proved by the appearance of extra visible absorption bands.

The third paper utilizes molecular modeling to innovate new peptidomimetic compounds to act as HCV NS3 protease antiviral. The suggested antivirals are divided into two groups. The first group has hexapeptide (Glu-Asp-Val-ValCys) binding to cellulose monomer at positions 2, 3 or 6 while the second group has hexapeptide (Glu-Asp-Val-ValCys-Cys) binding to cellulose dimmer at positions 2, 3, 6, 2', 3'or 6'. PM3 quantum mechanical method is utilized while higher level of theory at HF/3-21g**and B3LYP/3-21g** is utilized for verification. 